delivery of education workshops). Data from athletes' medical insurance scheme showed that the number of claims for mental health have increased and the average costs of these claims has decreased.

Conclusion Good start; high uptake in MHEP led to increasing their time; education programme built upon with $\mathrm{MH}$ Champions programme; possible correlation between increased awareness and earlier intervention, and increasing number of claims for mental health support with decreasing average cost; levels of psychological distress and wellbeing similar to levels observed in wider society.

\section{IMPLEMENTATION OF PSYCHIATRICI PSYCHOTHERAPEUTIC SUPPORT WITHIN A LONGITUDINAL HEALTH MONITORING IN COMPETITIVE PARA ATHLETES}

${ }^{1}$ Petra Dallmann, ${ }^{2}$ Rainer Leonhart, ${ }^{3}$ Anja Hirschmüller. 'Department of General Psychiatry, Center for Psychosocial Medicine, Heidelberg University Hospital, Heidelberg University, Heidelberg, Germany; ${ }^{2}$ University of Freiburg, Department of Psychology, Freiburg, Germany; ${ }^{3}$ Department of Orthopedics and Trauma Surgery, Medical Center - Albert-LudwigsUniversity of Freiburg, Faculty of Medicine, Albert-Ludwigs-University, Freiburg, Germany

\subsection{6/bjsports-2021-IOC.348}

Background Longitudinal monitoring of mental health symptoms in para athletes israre, so are strategies to prevent and detect mental illnesses in this group. Ideas to lower barriers for seeking for mental help are needed.

Objective Implementation of a mental health surveillance system, using the PHQ (Patient Health Questionnaire)-4 in highlevel Paralympic athletes in combination with a psychiatric/psychotherapeutic support which provided help either if athletes demanded or if PHQ-4 score exceeded 4 two weeks or longer.

Design Longitudinal weekly monitoring of depression and anxiety within the German National Paralympic Team accompanied by psychiatric/psychotherapeutic intervention.

Setting In preparation of the Paralympic Games in Tokyo, German athletes were invited to take part in a weekly, questionnaire-based monitoring program which included the PHQ-4 and level of stress.

Patients (or Participants) Data of 78 athletes was collected for one year (05/19-04/20). Within this group 21 athletes were contacted (8 male, 13 female, 8 individual sport, 13 team sport), 4 of them more than on one occasion.

Interventions (or Assessment of Risk Factors) Athletes were contacted via Email and offered support regarding mental health problems in case they requested help or if their PHQ-4 scores overreached the cut-off value of 4 in at least 2 consecutive weeks.

Main Outcome Measurements Number of athletes contacted because of PHQ-4, number of athletes demanding support and number of contacts which resulted in consultation during one year.

Results Twenty-one of 78 athletes were contacted, 11 times on demand, 13 times because of PHQ-4 scores. After the first contact psychiatric/psychotherapeutic support was requested 8 times, by 7 different athletes. This resulted in 7 phone calls and 1 email conversation. Seven of the 8 requests were on demand, whereas only one resulted from elevated PHQ-4 scores. Recommendations for sport psychology $(n=2)$, psychiatrist/psychotherapy $(n=4)$ were given six times. Help to find a local sport psychiatry/psychotherapy specialist was provided in 4 cases, questions concerning medication were answered in 1 case and support in an acute stress situation in 1 case.

Conclusions The demand for psychiatric/psychotherapeutic support was high ( $9 \%$ of all athletes within one year). Offering an easy access to psychiatric/psychotherapeutic help seems to be an effective way to support athletes concerning mental health issues.

\section{MAXIMISING THE RELEVANCE AND DISSEMINATION OF THE IOC MEDICAL CONSENSUS STATEMENTS: WHAT ARE THE CONSENSUS STATEMENTS AND HOW ARE THEY USED IN LITERATURE?}

${ }^{1}$ Caroline Finch, 'Lauren Fortington, ${ }^{4}$ Marelise Badenhorst, ${ }^{1}$ Rebecca Handcock, ${ }^{2}$ Evert Verhagen, ${ }^{5}$ Martin Schwellnus, ${ }^{3}$ Carolyn Emery, ${ }^{3}$ Kati Pasanen, ${ }^{4}$ Wayne Derman. ${ }^{1}$ Australian Centre for Research into Injury in Sport and its Prevention (ACRISP), School of Medical and Health Sciences, Edith Cowan University, Joondalup, Australia; ${ }^{2}$ Amsterdam Collaboration on Health and Safety in Sports, Department of Public and Occupational Health, Amsterdam UMC, Amsterdam, Netherlands; ${ }^{3}$ Sport Injury Prevention Research Centre, University of Calgary, Calgary, Canada; ${ }^{4}$ Insitute of Sport and Exercise Medicine, University of Stellenbosch, Stellenbosch, South Africa; ${ }^{5}$ Sport, Exercise Medicine and Lifestyle Institute (SEMLI), University of Pretoria, Pretoria, South Africa

\subsection{6/bjsports-2021-IOC.349}

Background The International Olympic Committee (IOC) Medical and Scientific Commission has a goal to provide guidance on athlete health for sports organisations. One strategy to meet this goal has been the development and publication of sports medicine consensus statements. It is currently unknown if there has been use of the consensus statements or if the overall goal of the statements - to improve athlete health and wellbeing - has been achieved.

Objective To identify and summarise citation measures of the IOC medical consensus statements.

Design Citation analysis.

Methods IOC medical consensus statements published from 2004 to 2018, and citing publications, were sourced from the IOC website, Scopus database and Google Scholar. Descriptive analyses over time of the number of consensus statements and citing documents with summaries of the authorship countries and keywords. Citation analyses were conducted to model links between consensus statements and citing publications, field weighted citation index (FWCI), and the SCImago Journal Ranking.

Results Twenty-seven consensus statements linked to the IOC medical and scientific commission were identified, addressing a range of topics from broad health and social issues to specific clinical topics. Authors from 30 countries contributed to the statements while citing papers were authored from 86 countries. Concussion was the most prominent key term in all citing documents. The youth athletic development statement has the highest FWCI (19.6), followed by concussion(18.8); load (12.3); relative energy deficiency(11.3); platelet-rich plasma (10.1); and supplements(9.9).

Conclusions Several consensus statements are widely used and cited in the literature while others have been less impactful through citation measures. The countries that use and cite 